\title{
Detection and Characterization of Fusarium oxysporum f. sp. vasinfectum VCG0114 (Race 4) Isolates of Diverse Geographic Origins
}

\author{
Alois A. Bell, ${ }^{1}$ Aixing Gu, ${ }^{2}$ Jim Olvey, ${ }^{3}$ Tanya A. Wagner, ${ }^{4}$ Javlon J. Tashpulatov, ${ }^{1}$ Sandria Prom, ${ }^{4}$ Jose Quintana, ${ }^{1}$ \\ Robert L. Nichols, ${ }^{5}$ and Jinggao Liu ${ }^{1, \dagger}$ \\ ${ }^{1}$ Southern Plains Agricultural Research Center, Agricultural Research Service, USDA, College Station, TX 77845 \\ ${ }^{2}$ Department of Plant Pathology, Xinjiang Agricultural University, Urumqi, China \\ ${ }^{3}$ O\&A Enterprises, Maricopa, AZ 85139 \\ ${ }^{4}$ Department of Plant Pathology and Microbiology, Texas A\&M University, College Station, TX 77845 \\ ${ }^{5}$ Cotton Incorporated, Cary, NC 27513
}

\begin{abstract}
A highly virulent cotton wilt pathogen, Fusarium oxysporum f. sp. vasinfectum VCG0114 (race 4) was found in West Texas in 2017, after being known in California since 2001. Isolates obtained from wilted plants collected in 2017 from Texas, in 2015 from China, and during 2001 to 2014 from California and isolates from historical collections including the race 4 reference isolate were characterized by soil-infestation pathogenicity assays, DNA sequence analysis, and vegetative compatibility analysis. All obtained $F$. oxysporum $\mathrm{f}$. sp. vasinfectum isolates belonged to VCG0114. All of these isolates, except one isolate from China, caused disease in a soil-infestation assay without nematodes. Thus, they belong to the nematode-independent pathotype. Texas isolates were significantly more virulent than were isolates from China or California on

detected among the genotypes in some locations. No differences in pathogenicity were observed between the California and China collection isolates on Pima S-7, and the virulence of the major genotypes was similar on the Gossypium hirsutum cultivar 'Stoneville 474' or the Barbren 713 germplasm line. Simple polymerase chain reaction (PCR) methods were developed to specifically determine and detect the four genotypes within VCG0114. A specific PCR method to detect all VCG0114 isolates was also developed. These methods will facilitate the timely identification of infested fields and seed lots and the elucidation of evolutionary relationships among the isolates. This should help to closely monitor the movement of the pathogen and reduce dissemination of these devastating pathogens.
\end{abstract} Gossypium barbadense 'Pima S-7'. Four different genotypes (N, T, MT, and MiT) were identified based on the transposable element Tfol insertion into the $P H O$ gene and independent MULE or MITE insertions into the Tfol transposon. Some significant differences in virulence were
Keywords: Fusarium oxysporum f. sp. vasinfectum, VCG0114, race 4, cotton, Fusarium wilt, Pima, Gossypium, Tfol, pathogen diversity, pathogen detection
Fusarium oxysporum Schlecht is a species complex composed of hundreds of genetically isolated populations that may show beneficial, commensal, or pathogenic relationships with plants (Di Pietro et al. 2003; Gordon and Martyn 1997). The pathogens vary in host specificity, environmental adaptation, and disease symptomology. Snyder and Hansen divided isolates that cause wilts or root rots into groups called formae speciales (f. sp.) based on host specificity (Snyder and Hansen 1940). Isolates attacking cotton were designated as f. sp. vasinfectum Atk. Sny. \& Hans, and F. oxysporum f. sp. vasinfectum occurs in most cotton (Gossypium spp.) growing regions of the world. Yield losses can be significant where soil conditions, nematode populations, and indigenous populations of the pathogen favor the disease.

${ }^{\dagger}$ Corresponding author: J. Liu; Jinggao.Liu@ars.usda.gov

The mention of firm names or trade products does not imply that they are endorsed or recommended by the United States Department of Agriculture (USDA) over other firms or similar products not mentioned. The USDA is an equal opportunity provider and employer.

Funding: The authors thank Cotton Incorporated (17-621) for their partial support of this research.

*The $\boldsymbol{e}$-Xtra logo stands for "electronic extra" and indicates that three supplementary tables are published online.

The author(s) declare no conflict of interest.

Accepted for publication 14 March 2019.

This article is in the public domain and not copyrightable. It may be freely reprinted with customary crediting of the source. The American Phytopathological Society, 2019.
Historically, variations in pathogenicity to diverse plant species as well as Gossypium species and cultivars were used to subgroup F. oxysporum f. sp. vasinfectum isolates into races 1 to 8 (Armstrong and Armstrong 1960, 1978; Chen et al. 1985; Ibrahim 1966). However, these race designations have been shown to be invalid as well as impractical (Bell et al. 2017; Davis et al. 1996; Holmes et al. 2009). In the present study, the mention of races is only meant to refer to the historical isolates. In recent years, isolates were genotypically characterized by DNA sequence and vegetative compatibility analyses in conjunction with pathogenicity assays (Abo et al. 2005; Bell et al. 2017; Cianchetta et al. 2015; Ortiz et al. 2017a; Skovgaard et al. 2001). More than 21 vegetative compatibility groups (VCGs) and two distinct pathotypes have been identified (Bell et al. 2017; Davis et al. 1996; Fernandez et al. 1994; Ortiz et al. 2017a). Isolates of VCG0113 (races 3 and 5), 0114 (races 4 and 7), and 01111 (Australian biotype) cause disease even in the absence of nematodes and are often associated with heavy clay or loam soils (Davis et al. 1996; Katan et al. 1983; Kim et al. 2005). This group of isolates cause extensive root damage and wilt in a soil-infestation assay but fail to cause wilt symptoms in a stem-puncture assay; thus, they are classified as a nematodeindependent, vascular-incompetent, or root-rot pathotype (Bell et al. 2016, 2017). Cotton host resistance to the fungus itself is needed to control the nematode-independent pathotype. Isolates of other VCGs are usually associated with sandy acidic soils and cause severe disease in the presence of the root-knot nematode Meloidogyne incognita (Chitwood) but inflict little to no damage when nematodes are absent in the field (Garber et al. 1979; Jorgenson et al. 1978). These isolates cause disease in the stem-puncture assay but not in the soilinfestation assay unless nematodes are added in the soil-infestation assay; therefore, they are classified as a vascular-competent or nematode-dependent pathotype (Bell et al. 2017). Breeding for resistance to root-knot nematode can reduce the incidence of the 
nematode-dependent pathotype (Wang and Roberts 2006). However, sting nematode (Belonolaimus longicaudatus) and reniform nematode (Rotylenchulus reniformis) also may affect wilt severity (Holdeman and Graham 1954; Neal 1954).

In the United States, only the nematode-dependent pathotype was recognized before 2001, with VCG0112 (race 2), 01117 (not race 1, but recognized as race 1 based on DNA similarity), and 01119 (race 8 ) as the most widely occurring genotypes (Bell et al. 2017; Ortiz et al. 2017a). Then in 2001, a highly virulent $F$. oxysporum f. sp. vasinfectum strain was identified as the causal agent inflicting severe wilt in Pima cotton (Gossypium barbadense) grown in heavy soils without nematodes in the San Joaquin Valley of California (Kim et al. 2005). The newly found strain was subsequently identified and designated as race 4 based on matches of partial sequences of translational elongation factor $(E F-1 \alpha)$, phosphate permease $(P H O)$, and $\beta$-tubulin $(B T)$ genes to those of the VCG0114 (race 4) reference isolate from India. The California isolates also vegetatively complemented the VCG0114 reference isolate (Ortiz et al. 2017a). The original VCG0114 from India was reported to infect cultivars of Gossypium arboreum but not those of G. barbadense and $G$. hirsutism (Armstrong and Armstrong 1960; Fahmy 1927; Kulkarni 1934). VCG0114 isolates from California also caused wilt on upland cotton (G. hirsutum) varieties but not as severely as on Pima cultivars (Kim et al. 2005). Race 7 isolates from China complemented VCG0114 isolates from India and California (Ortiz et al. 2017a), and their partial gene sequences of $E F-1 \alpha, P H O$, and $B T$ were identical to those of VCG0114 isolates from India and California (Kim et al. 2005; Skovgaard et al. 2001). Race 7 isolates caused disease in cultivars of G. hirsutum, G. barbadense, and G. arboreum (Chen et al. 1985). Isolates designated as "race 4-like" and the MDS-12 genotype were reported from Mid-South states (Cianchetta et al. 2015), and some of these isolates had DNA sequences of the partial $E F-1 \alpha$, $P H O$, and $B T$ genes identical to those of VCG0114 isolates. However, they are not vegetatively compatible with VCG0114 isolates (this study) and are nonpathogenic to cotton (Bell et al. 2017; Bennett et al. 2013).

The number of fields infested with VCG0114 has steadily increased within the San Joaquin Valley, and VCG0114 was also detected in the El Paso and Hudspeth Counties of Texas causing severe wilt of Pima cotton in 2017 (Halpern et al. 2018). Sequence analysis of the $\mathrm{PHO}$ gene revealed the presence of a $T f o l$ transposon insertion for some VCG0114 isolates and a second mutator-like transposon element (MULE) or miniature inverted-repeat transposable element (MITE) insertion within the inserted Tfol for others (Halpern et al. 2018; Ortiz et al. 2017a). All three of these transposable elements belong to class II (DNA) transposons that transpose themselves in the genome by a "cut and paste" mechanism (Feschotte and Pritham 2007; Lisch 2015; Raizada et al. 2001). They contain a central transposase coding region flanked by two terminal inverted repeats (TIRs). MITEs are shorter nonautonomous elements, missing the transposase domain, that can be mobilized by cognate transposases transcribed from other loci in the genome (Feschotte and Pritham 2007). Upon insertion, an 8-bp (Tfol) or a 9-bp (MULE and MITE) target site duplication (TSD) is generated. Thus, the insertion events observed in the $\mathrm{PHO}$ gene locus provide an opportunity to divide the VCG0114 isolates into four genotypes that otherwise are indistinguishable from each other. The present study was undertaken to genotype all VCG0114 and "race 4-like" MDS-12 isolates in our possession and to assess their corresponding pathogenicity with respect to genotype or geographical origin. The genotype determination was used to elucidate the historical movement of the pathogen and to define the baseline for future monitoring of the spread of the disease.

There are many possible mechanisms for the movement of the $F$. oxysporum f. sp. vasinfectum including seed-borne, insect and nematode transmission, and transmission by soil and plant debris movement (Bennett et al. 2008; Davis et al. 2006; Liu et al. 2011; Taubenhaus and Christenson 1936; Taubenhaus and Ezekiel 1932). These make containment of the organism problematic. Once established, the pathogen persists in the field for decades, even in the absence of the cotton host (Smith and Snyder 1975). Therefore, a reliable detection method is imperative for early identification of infested fields and seed lots to prevent further movement of VCG0114. The genotype determination methods can be utilized to detect the VCG0114 isolates that harbor transposable elements in the $P H O$ gene, which include most of the U.S. VCG0114 isolates. The polymerase chain reaction (PCR) detection of VCG0114 isolates that do not contain a transposon insertion in the $\mathrm{PHO}$ gene is problematic because of the sequence similarity in loci commonly used to identify $F$. oxysporum isolates, especially MDS- 12 isolates. The current detection methods for identifying these isolates require vegetative compatibility analysis coupled with the pathogenicity assay. Such an approach is labor intensive and time consuming. In this study, we report the development of a simple PCR method to specifically detect all VCG0114 isolates while discriminating against all non-VCG0114 isolates, including MDS-12 isolates. Differentiation is based on the unique Tfol insertion event in the promoter region of an $\mathrm{N}$-acetyltransferase (NAT) gene.

\section{Materials and Methods}

Plant materials and fungal isolates. In late May of 2017, symptomatic cotton plants were identified in two fields in El Paso County and one field in Hudspeth County of Texas. The samples were shipped overnight to the Insect Control and Cotton Disease Research Unit (ICCDRU), ARS-USDA, College Station, TX. In late July of 2017, symptomatic cotton plants were also collected in three other fields in El Paso County and another field in Hudspeth County and were brought to ICCDRU the next day. Upon receipt, lower stems and tap roots were dissected from the samples and surface sterilized by immersing in $70 \%$ ethanol for $3 \mathrm{~min}$ and rinsing with sterile water. The sterilized stems and roots were decorticated, trimmed, and immersed again in $70 \%$ ethanol for $1 \mathrm{~min}$, rinsed with sterile water, and cut into halves lengthwise. Each half was placed on Komada's Fusarium medium (Komada 1975). After 7 days, plates were observed under a dissecting microscope for the presence of $F$. oxysporum-like colonies and sporulation. Tentative $F$. oxysporum isolates were further purified by dissecting single-spore colonies from cultures growing on potato dextrose agar (PDA; Difco Laboratories, Detroit, MI) containing $100 \mathrm{ppm}$ of chloramphenicol and $50 \mathrm{ppm}$ of tetracycline (PDAA). Species were preliminarily identified according to Nelson et al. (1983) and subsequently confirmed by DNA sequencing (described below). These Texas isolates were designated with a TX prefix (TX1 to TX86) (Table 1).

Isolates from China's South and North Xinjiang regions were collected from late July to early August of 2015. Three-inch stem sections of wilted cotton plants from five counties were collected in the fields and allowed to dry quickly in brown paper bags before being shipped to ICCDRU. Upon receipt, the bottom $1 / 8$ inch and the top 1 inch of stem samples were trimmed off, and the resulting samples were processed as described for the Texas samples. These Chinese isolates were designated with a $\mathrm{CH}$ prefix $(\mathrm{CH} 1869$ to $\mathrm{CH} 1967$; Table 1). MDS-12 genotype isolates (Cianchetta et al. 2015) (AL28A, AL13C, AR13, AR13D LA1E, and MS15) were kindly provided by H. K. Doan and R. M. Davis (Department of Plant Pathology, University of California, Davis). All other reference isolates were described in Ortiz et al. (2017a) and Bell et al. (2017). Cultures from single spores were grown on PDAA or sorghum seeds, dried, and then stored at $30^{\circ} \mathrm{C}$

Vegetative compatibility analysis. Vegetative compatibilities of the Texas, China, and MDS-12 isolates were tested according to the method of Bell et al. (2017). A few of the Texas and China isolates and all the MDS-12 isolates failed to complement any known VCG and failed to cause disease in cotton (see below). They were not assigned an international code as suggested by Katan and Di Primo (1999) but designated with a representative isolate number preceded by letters NP (Table 1). For example, CH1891 belongs to VCGNP1977.

DNA extraction, sequencing, and phylogenetic analysis. Mycelium preparation and DNA extraction were performed according to the method of Bell et al. (2017). DNA concentrations were 
determined via Nanodrop (Thermo Scientific, Wilmington, DE), and integrity was analyzed by gel electrophoresis.

Representative isolates of each genotype that had not been sequenced previously were selected for sequencing. Portions of three nuclear genes $(E F-1 \alpha, P H O$, and $B T)$ were amplified according to the method of Ortiz et al. (2017a). PHO sequences with Tfol, Tfol/MITE, or Tfol/MULE insertions (Fig. 1) were amplified with primers $\mathrm{PHO} 48 \mathrm{~F}$ and $\mathrm{PHO} 2034$. The NAT gene with a Tfol insertion (Fig. 1) in its promoter was amplified with primers $\mathrm{Tfo} 828 \mathrm{~F}$ and NAT2888R (Ortiz et al. 2017a). Amplification was carried out in $50-\mu 1$ reaction mixtures with $50 \mathrm{ng}$ of template DNA, $1.75 \mathrm{mM}$ $\mathrm{MgCl}_{2}, 350 \mu \mathrm{M}$ dNTPs, $300 \mathrm{nM}$ each primer, and $3.75 \mathrm{U}$ of Expand Long Template Enzyme mix in 1× Expand Long Template Buffer 1 (Roche, Mannheim, Germany) and performed in an Eppendorf Mastercycler (Eppendorf, Westbury, NY) with the following program: initial denaturing at $94^{\circ} \mathrm{C}$ for $2 \mathrm{~min}, 35$ cycles of denaturing at $94^{\circ} \mathrm{C}$ for $20 \mathrm{~s}$, annealing at $55^{\circ} \mathrm{C}$ for $30 \mathrm{~s}$, extension at $68^{\circ} \mathrm{C}$ for $5 \mathrm{~min}$ for Tfol or Tfol/MITE insertions and $7 \mathrm{~min}$ for Tfol/MULE insertion, and a final extension at $68^{\circ} \mathrm{C}$ for $7 \mathrm{~min}$. An aliquot of PCR products was checked for target amplicons using gel electrophoresis. Additional internal primers in Supplementary Table S3 and in Ortiz et al. (2017a) were used for sequencing the whole amplicons. The PCR products were sequenced through MCLAB (South San Francisco, CA) by Sanger sequencing and were analyzed with a 3730XL DNA Analyzer. Sequences were edited and aligned using Sequencher version 5.4.6 (Gene Codes, Ann Arbor, MI). Sequences were deposited in GenBank with the accession numbers given in Supplementary Table S2.

For phylogenetic analysis, DNA sequences were aligned using the Clustal W program (Thompson et al. 1994) and adjusted manually. The maximum likelihood phylogenetic tree was constructed using
IQtree 1.6.9 (Nguyen et al. 2015) based on concatenated sequences of $E F-1 \alpha, P H O$, and $B T$ genes of the selected isolates. Partition models in edge-linked fashion (Chernomor et al. 2016) were used to partition the three genes in the concatenated sequences and to find the respective optimal substitution model (Kalyaanamoorthy et al. 2017) for each partition. Branch supports for the phylogenetic tree were estimated using ultrafast bootstrap approximation (Hoang et al. 2018). The $T f o l$ insertion sequence was coded as a single character, and additional insertions of MITE or MULE were coded as other single different characters in the $\mathrm{PHO}$ sequence. Sequences of $F$. fujikuroi isolate TX75 and F. verticillioides strain 7600 (NCBI genome assembly accession number: GCA_000149555.1) were included to root the tree.

Genotype determination and detection of VCG0114 isolates. VCG0114 isolates were genotyped based on the presence of Tfol (T type), Tfol/MULE (MT type), Tfol/MITE (MiT type), or null (N type) in the $\mathrm{PHO}$ gene (Fig. 1). They were further divided according to their geographic origins. For example, $\mathrm{T}$ type from Texas was designated as TTx and that from California as TCa. See footnote $\mathrm{x}$ of Table 1 for the complete set of designations. T, MiT, and $\mathrm{N}$ genotypes were determined by a modified multiplex PCR detection method (Ortiz et al. 2017a) with primers FovP-F, FovT-R, and FovP-R. Amplification in 20- $\mu$ l reaction mixture containing $20 \mathrm{ng}$ of template DNA, $2.5 \mathrm{mM} \mathrm{MgCl} 2,200 \mu \mathrm{M}$ dNTP, $200 \mathrm{nM}$ each primer, and $0.5 \mathrm{U}$ of GoTaq DNA polymerase in $1 \times$ GoTaq Flexi Buffer (Promega Corp., Madison, WI) was performed in an Eppendorf Mastercycler with the following program: initial denaturing at $94^{\circ} \mathrm{C}$ for $2 \mathrm{~min} ; 35$ cycles of denaturing at $94^{\circ} \mathrm{C}$ for $30 \mathrm{~s}$, annealing at $58^{\circ} \mathrm{C}$ for $30 \mathrm{~s}$, and extension at $72^{\circ} \mathrm{C}$ for $40 \mathrm{~s}$; and final extension at $72^{\circ} \mathrm{C}$ for $5 \mathrm{~min}$. The MT genotype was determined using the same PCR detection method except the primers were replaced with primer

Table 1. Genotype determination and specific polymerase chain reaction (PCR) detection of Fusarium oxysporum f. sp. vasinfectum VCG0114 (race 4) isolates of diverse geographic origins ${ }^{\vee}$

\begin{tabular}{|c|c|c|c|c|c|c|c|c|}
\hline \multirow[b]{2}{*}{ No. of isolates } & \multirow[b]{2}{*}{ VCG $^{\mathbf{w}}$} & \multirow[b]{2}{*}{ Type $^{x}$} & \multirow[b]{2}{*}{ Origin } & \multirow[b]{2}{*}{ Collection year } & \multicolumn{4}{|c|}{$\mathbf{P C R}^{\mathbf{y}}$} \\
\hline & & & & & $\begin{array}{l}\text { FovT-A } \\
(500 \text { bp) }\end{array}$ & $\begin{array}{l}\text { FovP-M } \\
\text { (426 bp) }\end{array}$ & $\begin{array}{l}\text { FovP-T } \\
(583 \text { bp })\end{array}$ & $\begin{array}{c}\text { FovP-P } \\
\text { (396 bp) }\end{array}$ \\
\hline \multicolumn{9}{|c|}{ VCG0114, F. oxysporum f. sp. vasinfectum } \\
\hline 28 & 0114 & MTTx & El Paso Co., TX & 2017 & + & + & - & - \\
\hline 8 & 0114 & MTTx & Hudspeth Co., TX & 2017 & + & + & - & - \\
\hline 30 & 0114 & TTx & El Paso Co., TX & 2017 & + & - & + & - \\
\hline 16 & 0114 & $\mathrm{NCh}$ & Xinjiang, China & 2015 & + & - & - & + \\
\hline 2 & 0114 & MTCh & Xinjiang, China & 2015 & + & + & - & - \\
\hline 1 & 0114 & NChA & Xinjiang, China & 2015 & - & - & - & + \\
\hline 2 & 0114 & $\mathrm{NChN}$ & China & $1985<>2001$ & + & - & - & + \\
\hline 15 & 0114 & $\mathrm{TCa}$ & California & 2001-2014 & + & - & + & - \\
\hline 1 & 0114 & MiTCa & California & 2014 & + & - & $t^{\mathrm{z}}$ & - \\
\hline 1 & 0114 & $\mathrm{NCa}$ & California & 2014 & + & - & - & + \\
\hline 6 & 0114 & NIn & India & $1936<>2001$ & + & - & - & + \\
\hline \multicolumn{9}{|c|}{ Other VCGs, $F$. oxysporum $\mathrm{f}$. sp. vasinfectum } \\
\hline 28 & 16 VCGs & $\ldots$ & Various & Various & - & - & - & + \\
\hline \multicolumn{9}{|c|}{ Nonpathogenic $F$. oxysporum from cotton } \\
\hline 8 & $\ldots$ & MDS-12 & Mid-South U.S.A. & $2011-2015$ & - & - & - & + \\
\hline 3 & $\ldots$ & $\ldots$ & West Texas & 2017 & - & - & - & + \\
\hline 4 & NP1977 & $\ldots$ & Xinjiang, China & 2015 & - & - & - & + \\
\hline \multicolumn{9}{|c|}{ Nonpathogenic F. fujikuroi from cotton } \\
\hline 1 & $\ldots$ & $\ldots$ & El Paso Co., TX & 2017 & - & - & - & + \\
\hline
\end{tabular}

${ }^{v}$ For information on individual isolates included in this table, see the supplementary materials.

${ }^{\mathrm{w}} \mathrm{VCG}=$ vegetative compatibility group.

x Type: genotypes within VCG0114 were designated based on the presence of transposable elements within the $P H O$ gene in conjunction with their geographic origins. MTTx $=$ mutator-like transposable element $(M U L E)$ within Tfol transposon/Texas; TTx $=$ Tfol transposon/Texas; $\mathrm{NCh}=$ no transposon/China; $\mathrm{MTCh}=$ mutator like transposable element within $T f o l$ transposon/China; $\mathrm{NChA}=$ no transposon/China-negative for Tfol in the promoter region of NAT gene; NChN = no transposon/China (NRRL collection); TCa $=$ Tfol transposon/California; $\mathrm{MiTCa}=$ miniature inverted-repeat transposable element $(M I T E)$ within Tfol transposon/California; $\mathrm{NCa}=$ no transposon/California; and $\mathrm{NIn}=$ no transposon/India.

y FovT-A = detected with primers FovT-F (within Tfol) and FovA-R (within promoter region of NAT); FovP-M = detected with primers FovP-F (within PHO) and FovM-R (within MULE); FovP-T = detected with primers FovP-F and FovT-R (within Tfol); FovP-P = detected with primers FovP-F and FovP-R (within PHO); and + denotes presence of the band and - denotes absence of the band. Primers are shown in the supplementary materials.

$\mathrm{z}$ The presence of the 71-bp MITE within the $5^{\prime}$ end of Tfol transposon in isolate MD308 gives a 663-bp band instead of a 583-bp band (71 bp MITE + 9 bp TSD + $583 \mathrm{bp}=663 \mathrm{bp})$. 
pair FovP-F and FovM-R. Alternatively, all four genotypes can be detected in a single multiplex PCR with a primer mixture of FovPF, FovM-R, FovT-R, and FovP-R using the same PCR program as above. Isolates from other VCGs of $F$. oxysporum $\mathrm{f}$. sp. vasinfectum and from cotton-associated nonpathogenic $F$. oxysporum and $F$. fujikuroi were also tested to evaluate specificity of these PCR tests.

Development of a VCG0114-specific PCR detection method. 5' terminal inverted repeat (TIR) and $3^{\prime}$ TIR sequences of Tfol were used to BLAST search the genome of VCG0114 isolate NRRL25433 (NCBI genome accession no. GCA_000260175.2). Sequences flanking Tfols were used to design primers. Forward primers flanking the $5^{\prime}$ TIR were paired with FovT-R, and reverse primers flanking the $3^{\prime}$ TIR were paired with FovT-F to PCR screen one or two isolates of each genotype listed in Table 1 using the PCR conditions described above. The primer FovA-R, when paired with FovT-R, produced a positive band only for the VCG0114 isolates in the PCR screen. This PCR test was evaluated using all the isolates listed in the supplementary materials. If no band was observed (negative) for an isolate in the test, the test for the isolate was repeated with a primer mixture of the original primer pair and the primer pair EF1100F and EF1885R (Ortiz et al. 2017a), which was added as an internal positive control.

Soil-infestation assays. Pathogenicity tests of VCG0114 isolates were conducted on G. barbadense 'Pima S-7', G. hirsutum 'Stoneville 474' (ST 474), and G. hirsutum germplasm line Barbren 713 (Bell et al. 2015). Inoculum was prepared by flooding 7-day-old PDAA cultures with sterile water, dislodging the conidia from the plate with a glass rod, and filtering the resulting conidial suspension through two layers of Kimwipes (Kimberly-Clark, Roswell, GA) Conidia concentration was determined with a hemocytometer, and the filtrate was adjusted to $5 \times 10^{6}$ conidia $/ \mathrm{ml}$ by adding water.

The following isolates were used to inoculate Pima S-7 (G. barbadense): TX1 to 36 for the MTTx genotype, TX37 to 51 and 58 to 74 for the TTx genotype; CH1869, 1889, 1907 to 1909, 1911 to 1913, 1934, 1944, 1960, 1965, 1969, 1970, 1973, and 1975 for the NCh genotype; CH1971 and 1972 for the MTCh genotype; CH1967 for NChA; CA9, 14, MD305 to 307, 309, 310, and 313 to 317 for the TCa genotype; MD308 for the MiTCa genotype; MD312 for the NCa genotype; TX76, 84, and 85 for nonpathogenic $F$. oxysporum; and TX75 for nonpathogenic F. fujikuroi (Table 2). Assay soils were prepared by mixing $30 \mathrm{~kg}$ of sandy loam, $10 \mathrm{~kg}$ of washed sand, and $80 \mathrm{~g}$ of fine-ground oatmeal. The mixture was pasteurized and fertilized as described in Bell et al. (2017). Pima S-7 seeds were pregerminated according to the method of Bell et al. (2017). Seedlings with a radicle length of about 2 to $3 \mathrm{~cm}$ were transplanted to the prepared $500-\mathrm{ml}$ soil cups, one plant per cup. One milliliter of $F$. oxysporum f. sp. vasinfectum inoculum was injected into each of six locations equidistant from each other and $1 \mathrm{~cm}$ away from the radicle with an 18-gauge needle retracted from a depth of $4 \mathrm{~cm}$ to the soil surface during injection. Three plants were inoculated with each isolate. Ten control plants were inoculated with the same volume of sterile water. The plants were grown in a growth chamber at $23 / 18^{\circ} \mathrm{C}(13 \mathrm{~h} \mathrm{light/}$ $11 \mathrm{~h}$ dark) and fertilized weekly with $150 \mathrm{mg}$ of Peter's Peat-Lite Special 10:30:20 containing chelated minor elements. After 38 days, the number of wilted true leaves including the true-leaf scars, the total
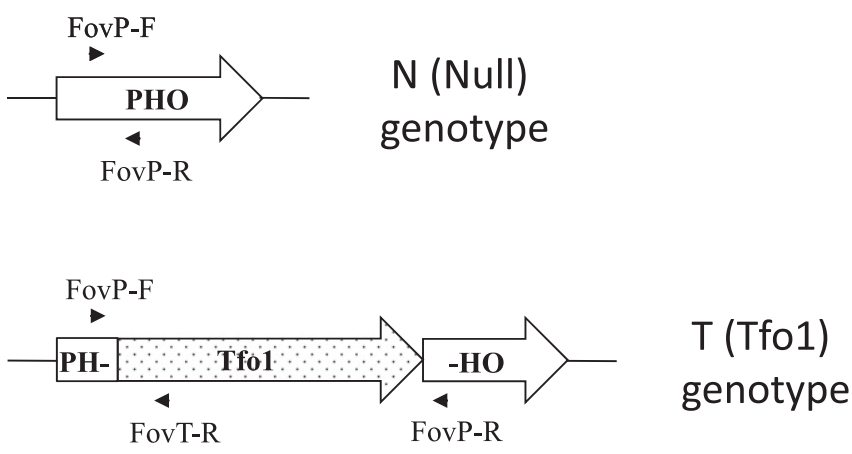

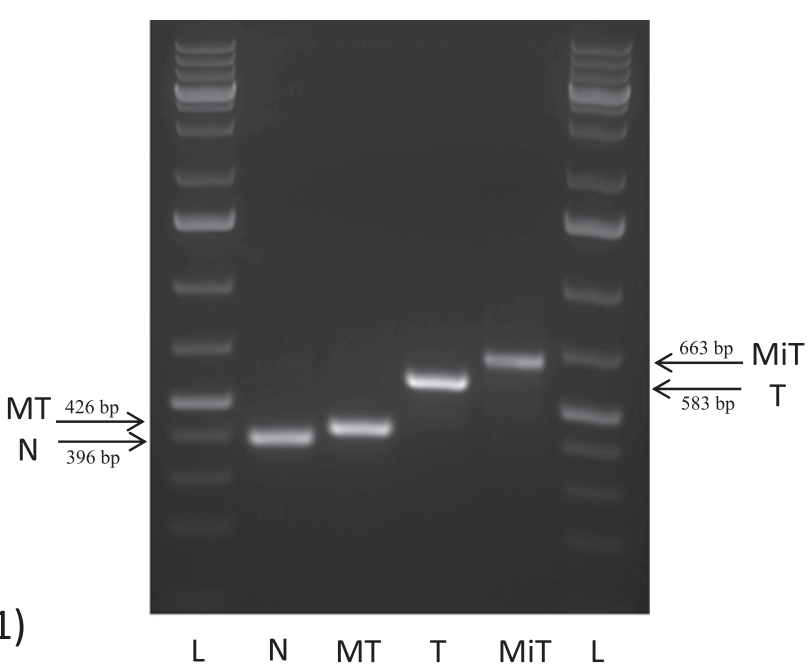

MiT (MITE/Tfo1) genotype

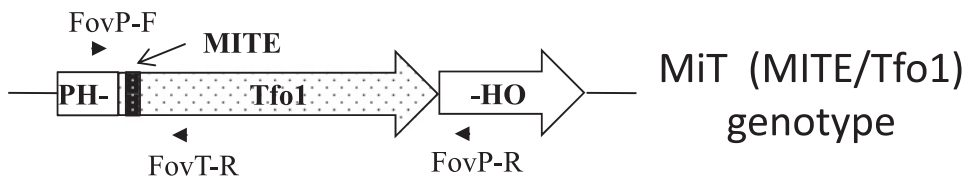

L $\quad \mathrm{N} \quad \mathrm{MT} \quad \mathrm{T} \quad$ MiT $\mathrm{L}$

MT (MULE/Tfo1) genotype
VCG0114 genotype

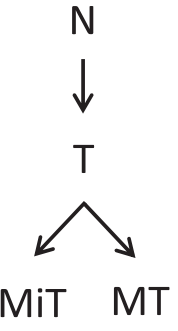

Fig. 1. Genotype determinations of Fusarium oxysporum f. sp. vasinfectum VCG0114 isolates and their specific detection. Left panel: genotypes observed among VCG0114 isolates based on the various transposable element insertion events at the PHO locus and their specific detections. The genotype of VCG0114 is determined and specifically detected based on the unique Tfo1 insertion event at the N-acetyltransferase gene (NAT) locus using a primer pair FovT-F and FovA-R with an amplicon size of 500 bp. The $\mathrm{N}$ (null) genotype determination is not specific unless the VCG0114 genotype determination is positive at the NAT locus. Target locations of polymerase chain reaction (PCR) primers are indicated on the diagrams. Right panel top: examples of genotype determination by a multiplex PCR with a primer mixture of FovP-F, FovP-R, FovT-R, and FovM-R. Lane L = 1-kb ladder DNA plus size marker; lane N = N genotype, Indian isolate ATCC16613; lane MT = MULE/Tfo1 genotype, Texas isolate TX11; lane T = Tfo1 genotype, Texas isolate TX39; and lane MiT = MITE/Tf01 genotype, California isolate MD308. Right panel bottom: proposed evolutionary relationships of the VCG0114 genotypes. 
number of true leaves including the true-leaf scars, fresh shoot weight, shoot height, and stem section vascular browning index were measured. The percentage of wilted leaves was calculated as follows: (number of wilted true leaves including the true-leaf scars)/(total number of true leaves including the true-leaf scars) $\times 100$. The stem sections just above the soil line were evaluated for vascular staining using the following vascular staining index: $0=$ no staining; $1=0$ to $10 \%$ of cross section area stained; $2=10$ to $30 \%$ of area stained; $3=$ 30 to $70 \%$ of area stained; $4=70$ to $90 \%$ of area stained; $5=90$ to $100 \%$ of area stained or the plant was dead. A 1-inch stem section just above soil line was then cut, immersed in $70 \%$ ethanol for $3 \mathrm{~min}$, decorticated, rinsed with sterile water, and plated on Komada's Fusarium medium. After 7 days, emergence of Fusarium from sections was recorded. At least several isolates within each genotype were subjected to PCR genotype determination described above to confirm recovery of inoculum. The whole experiment was considered as one trial, and it was repeated four times in total (four trials).

ST 474 (G. hirsutum) plants were inoculated with following isolates: CH1869, 1889, 1907 to 1909,1911 to 1913,1934 , $1944,1960,1965,1969,1970,1973$, and 1975 for the NCh genotype; $\mathrm{CH} 1971$ and 1972 for the MTCh genotype; $\mathrm{CH} 1967$ for NChA; NRRL25433 and 31672 for NChN; CA9, 12, 14, S9, MD305 to 307, 309 to 311 , and 313 to 317 for the TCa genotype; MD308 for MiTCa; MD312 for NCa; ATCC16613, BBA69519, 69520, 69521, NRRL31669, and 31673 for NIn; CH1891, 1974, 1977, and 1981 for nonpathogenic $F$. oxysporum from China; and AR13 for a nonpathogenic $F$. oxysporum of MDS-12 genotype (Table 3). The inoculation and disease evaluation procedures were the same as with Pima S-7 assays except that five plants were treated with each isolate and the plants were evaluated after 5 weeks of growth in the growth chambers. The whole experiment was considered as one trial, and it was repeated three times in total (three trials).

Barbren 713 (G. hirsutum) plants were inoculated with following isolates: CH1869, 1889, 1907 to 1909,1911 to $1913,1934,1944$, 1960, 1965, 1969, 1970, 1973, and 1975 for the NCh genotype;
CH1971 and 1972 for the MTCh genotype; CH1967 for NChA; CA9, 14, MD305 to 307,309 to 311 , and 313 to 317 for the TCa genotype; MD308 for MiTCa; and MD312 for NCa (Table 4). The inoculation and disease evaluation procedures were the same as with ST 474 assays except that the plants were evaluated after 6 weeks of growth in the growth chambers. The whole experiment was considered as one trial, and it was repeated two times in total (two trials).

Statistical analysis. Data from response variables of the soil infestation with $F$. oxysporum $\mathrm{f}$. sp. vasinfectum isolates were modeled separately for each of the three cotton varieties or line using a mixed-models approach (PROC GLIMMIX; SAS version 9.4, SAS Institute, Cary, NC). Each trial for each host was a completely randomized design. We modeled each response variable on the whole data for each host with genotype-origin as a fixed effect. Random effects included trial, isolate within each genotype-origin nested in trial, and replication nested within the isolate. Studentized marginal and conditional residuals were plotted to verify that residuals were independent and normally distributed. Means were separated at $P=0.05$ using least squares means with the Tukey-Kramer adjustment.

\section{Results}

F. oxysporum f. sp. vasinfectum isolates from 2015 Xinjiang sample collection. Stem samples of wilted cotton plants collected in fields of Southern and Northern Xinjiang, China, in 2015 yielded a total of $23 F$. oxysporum isolates. Vegetative compatibility analysis confirmed that 19 of these belonged to $F$. oxysporum f. sp. vasinfectum VCG0114 (Table 1). The other four isolates complemented each other but not any known $F$. oxysporum $\mathrm{f}$. sp. vasinfectum $\mathrm{VCG}$ or any nonpathogenic $F$. oxysporum VCG in our collection. Phylogenetic analysis placed these isolates on clade III as a new genotype of cotton-associated $F$. oxysporum (Fig. 2). Pathogenicity assays (see below) showed that they were nonpathogenic to cotton. Therefore, they were designated as VCGNP1977 (Table 1).

The duplex PCR test for Tfol insertion in PHO (Ortiz et al. 2017a) for the 19 VCG0114 isolates confirmed that 17 of them lacked the

Table 2. Fresh shoot weight, shoot height, percent leaves wilted, and vascular browning index of Gossypium barbadense Pima S-7 after 38 days of growth in soils infested with isolates of different genotypes within VCG0114 of Fusarium oxysporum f. sp. vasinfectum from diverse geographic origins or nonpathogenic F. oxysporum isolates associated with cotton ${ }^{\mathrm{t}}$

\begin{tabular}{|c|c|c|c|c|c|}
\hline Type $^{u}$ & No. of isolates & Shoot weight (g) & Shoot height $(\mathrm{cm})$ & Percent leaves wilted ${ }^{v}$ & Vascular browning index ${ }^{w}$ \\
\hline MTTx & 36 & $1.0(94.9)^{\mathrm{x}} \mathrm{a}$ & $7.6(77.7)^{x} a$ & $91.8 \mathrm{a}$ & $94.4 \mathrm{a}$ \\
\hline TTx & 30 & $1.6(91.8) \mathrm{a}$ & $9.3(72.7) a b$ & $92.7 \mathrm{a}$ & $92.9 \mathrm{a}$ \\
\hline $\mathrm{NPF}^{\mathrm{y}}$ & 4 & $15.7(19.5) \mathrm{c}$ & $29.6(13.2) \mathrm{d}$ & $0.4 \mathrm{e}$ & $3.0 \mathrm{e}$ \\
\hline $\mathrm{NCh}$ & 16 & $5.0(74.4) b$ & $15.3(55.1) \mathrm{c}$ & $75.5 \mathrm{ab}$ & $78.0 \mathrm{~b}$ \\
\hline MTCh & 2 & $7.2(63.1) b$ & $16.9(50.4) \mathrm{c}$ & $56.7 \mathrm{bd}$ & $55.2 \mathrm{~cd}$ \\
\hline NChA & 1 & $17.3(11.3) \mathrm{c}$ & $29.1(14.7) \mathrm{d}$ & $0.0 \mathrm{e}$ & $0.0 \mathrm{e}$ \\
\hline $\mathrm{TCa}$ & 12 & $5.2(73.3) b$ & $13.6(60.1) b c$ & $68.9 \mathrm{bc}$ & $71.7 \mathrm{bd}$ \\
\hline MiTCa & 1 & $1.7(91.3) \mathrm{ab}$ & 8.7 (74.5) ac & 83.7 acd & $89.8 \mathrm{abc}$ \\
\hline $\mathrm{NCa}$ & 1 & 4.9 (74.9) ab & $12.5(63.3) \mathrm{ac}$ & 78.7 acd & $74.8 \mathrm{abc}$ \\
\hline Control $^{\mathrm{z}}$ & 0 & $19.5 \mathrm{c}$ & $34.1 \mathrm{~d}$ & $3.2 \mathrm{e}$ & $1.8 \mathrm{e}$ \\
\hline
\end{tabular}

$\mathrm{t}$ Values are the least squares means of the responses treated by the number of isolates indicated in the second column for each genotype of VCG0114 of $F$. oxysporum f. sp. vasinfectum with three replicates for each isolate in each of the four trials. Means were separated at $P=0.05$ using least squares means with the Tukey-Kramer adjustment. Means with the same letter within the column are not significantly different. The following isolates were used: TX1 to 36 for MTTx; TX37 to 51 and 58 to 74 for TTx; CH1869, 1889, 1907 to 1909, 1911 to 1913, 1934, 1944, 1960, 1965, 1969, 1970, 1973, and 1975 for NCh; CH1971 and 1972 for MTCh; CH1967 for NChA; CA9, 14, MD305 to 307, 309, 310, and 313 to 317 for TCa; MD308 for MiTCa; and MD312 for NCa.

u Type: genotypes within VCG0114 were designated based on the presence of transposable elements within the $P H O$ gene in conjunction with their geographic origins. MTTx = mutator-like transposable element $(M U L E)$ within Tfol transposon/Texas; TTx = Tfol transposon/Texas; NCh = no transposon/China; MTCh = MULE within Tfol transposon/China; NChA = no transposon/China-negative for Tfol insertion in the promoter region of NAT; TCa = Tfol transposon/California; MiTCa = miniature inverted-repeat transposable element $(M I T E)$ within Tfol transposon/California; and NCa = no transposon/California.

$\checkmark$ Percentage of leaves wilted was calculated by dividing the number of wilted leaves including the leaf scars by the total number of leaves including the leaf scars within a wilted plant multiplied by 100 .

w Vascular browning index was initially assigned with the following scale: $0=$ no staining; $1=0$ to $10 \%$ of cross section stained; $2=10$ to $30 \%$ of area stained; $3=$ 30 to $70 \%$ of area stained; $4=70$ to $90 \%$ of area stained; and $5=90$ to $100 \%$ of area stained or the plant was dead. For ANOVA-type statistical analysis, they were converted to percentage basis by taking the midpoint for each scale: scale 0 became $0 \%, 1$ became $5 \%, 2$ became $20 \%, 3$ became $50 \%$, 4 became $80 \%$, and 5 became $95 \%$.

$x$ Percent reduction compared with control is given in parentheses.

y Three nonpathogenic F. oxysporum isolates (TX76, 84, and 85) and one nonpathogenic F. fujikuroi isolate (TX75) from 2017 Texas collection were also included as controls.

z Controls consisted of plants inoculated with water with 10 replicates in each of the four trials. 
insertion (N genotype in Figure 1, left panel). Sequence analysis of $E F$ 1- $\alpha, P H O$, and $B T$ genes of these 17 isolates confirmed that all had identical sequences with that of the reference VCG0114 isolate ATCC16613. The remaining two isolates, $\mathrm{CH} 1971$ and 1972, had $E F$ 1- $\alpha$ and $B T$ sequences identical to those of isolate ATCC16613 but failed to amplify the Tfol insertion or the noninsertion band.
Amplification with the primer pair FovT-F and FovP-R (MT [MULE/ Tfol] genotype in Figure 1, left panel) for the $3^{\prime}$ end of the Tfol/PHO insertion junction yielded the expected 742-bp band for both isolates, indicating a possible second transposable element insertion within the inserted Tfol. Amplification with primers PHO48F and PHO2034 (Ortiz et al. 2017a) and a long template Taq and

Table 3. Fresh shoot weight, shoot height, percent leaves wilted, and vascular browning index of Gossypium hirsutum 'Stoneville 474 ' after 35 days of growth in soils infested with isolates of different subgenotypes within VCG0114 of Fusarium oxysporum f. sp. vasinfectum from diverse geographic origins or nonpathogenic $F$. oxysporum and $F$. fujikuroi isolates associated with cotton ${ }^{\text {s }}$

\begin{tabular}{|c|c|c|c|c|c|}
\hline Type $^{t}$ & No. of isolates & Shoot weight (g) & Shoot height (cm) & Percent leaves wilted ${ }^{u}$ & Vascular browning index $x^{v}$ \\
\hline $\mathrm{NCh}$ & 16 & $9.4(26.6)^{\mathrm{w}}$ bd & $21.1(19.8)^{\mathrm{w}} \mathrm{b}$ & $46.6 \mathrm{~b}$ & $43.0 \mathrm{~b}$ \\
\hline MTCh & 2 & $2.4(81.3) \mathrm{a}$ & $10.2(61.2) \mathrm{a}$ & $80.8 \mathrm{a}$ & $86.4 \mathrm{a}$ \\
\hline NChA & 1 & $13.3(-3.9)$ cde & 25.2 (4.2) bcef & $9.2 \mathrm{de}$ & 9.9 cef \\
\hline $\mathrm{NPF}^{\mathrm{x}}$ & 4 & $13.0(-1.6)$ ce & $27.9(-6.1)$ ef & $6.8 \mathrm{e}$ & 7.9 ce \\
\hline $\mathrm{NChN}$ & 2 & $12.9(-0.8)$ cde & $26.1(0.8) \mathrm{def}$ & $0.0 \mathrm{e}$ & $0.0 \mathrm{e}$ \\
\hline $\mathrm{TCa}$ & 15 & $10.2(20.3) b c$ & $21.7(17.5) \mathrm{bc}$ & $29.5 \mathrm{~cd}$ & 32.9 bdf \\
\hline MiTCa & 1 & $8.7(32.0) b c$ & $20.2(23.2) \mathrm{bd}$ & 25.1 bce & $41.0 \mathrm{bc}$ \\
\hline $\mathrm{NCa}$ & 1 & 10.7 (16.4) be & 22.4 (14.8) be & 25.6 bce & $29.3 \mathrm{bf}$ \\
\hline NIn & 6 & $14.5(-13.3) \mathrm{e}$ & $28.7(-9.1) \mathrm{f}$ & $1.7 \mathrm{e}$ & $0.0 \mathrm{e}$ \\
\hline $\operatorname{MDS}^{y}$ & 1 & $13.2(-3.1)$ cde & 25.3 (3.8) bcef & $9.2 \mathrm{de}$ & 9.9 cde \\
\hline Control $^{\mathrm{z}}$ & 0 & $12.8 \mathrm{cde}$ & $26.3 \mathrm{cdef}$ & $0.9 \mathrm{e}$ & $0.8 \mathrm{e}$ \\
\hline
\end{tabular}

$\mathrm{s}$ Values are the least squares means of the responses treated by the number of isolates indicated in the second column for each genotype of VCG0114 of F. oxysporum f. sp. vasinfectum with five replicates for each isolate in each of the three trials. Means were separated at $P=0.05$ using least squares means with the Tukey-Kramer adjustment. Means with the same letter within the column are not significantly different. The following isolates were used: $\mathrm{CH} 1869,1889$, 1907 to 1909,1911 to 1913, 1934, 1944, 1960, 1965, 1969, 1970, 1973, and 1975 for NCh; CH1971 and 1972 for MTCh; CH1967 for NChA; NRRL25433 and 31672 for NChN; CA9, 12, 14, S9, MD305 to 307, 309 to 311, and 313 to 317 for TCa; MD308 for MiTCa; MD312 for NCa; and ATCC16613, BBA69519, 69520, 69521, NRRL31669, and 31673 for NIn.

t Type: genotypes within VCG0114 were designated based on the presence of transposable elements within the PHO gene in conjunction with their geographic origins. $\mathrm{NCh}=$ no transposon/China; MTCh = mutator-like transposable element $(M U L E)$ within $T f o l$ transposon/China; NChA = no transposon/Chinanegative for Tfol insertion in the promoter region of $N A T ; \mathrm{NChN}=$ no transposon/China (NRRL collection); $\mathrm{TCa}=T f o 1$ transposon/California; $\mathrm{MiTCa}=\mathrm{miniature}$ inverted-repeat transposable element $(M I T E)$ within $T f o 1$ transposon/California; $\mathrm{NCa}=$ no transposon/California; and $\mathrm{NIn}=$ no transposon/India .

" Percentage of leaves wilted was calculated by dividing the number of wilted leaves including the leaf scars by the total number of leaves including the leaf scars within a wilted plant multiplied by 100 .

$\checkmark$ Vascular browning index was initially assigned with the following scale: $0=$ no staining; $1=0$ to $10 \%$ of cross section stained; $2=10$ to $30 \%$ of area stained; $3=$ 30 to $70 \%$ of area stained; $4=70$ to $90 \%$ of area stained; and $5=90$ to $100 \%$ of area stained or the plant was dead. For ANOVA-type statistical analysis, they were converted to percentage basis by taking the midpoint for each scale: scale 0 became $0 \%, 1$ became $5 \%, 2$ became $20 \%, 3$ became $50 \%, 4$ became $80 \%$, and 5 became $95 \%$.

w Percent reduction compared with control is given in parentheses.

x Four nonpathogenic VCGNP1977 F. oxysporum isolates (CH1891, 1974, 1977, and 1981), isolated from the 2015 China collection.

y $F$. oxysporum isolate AR13, from U.S. Mid-South group (MDS-12), which had $E F-1 \alpha, P H O$, and $B T$ gene sequences identical to that of VCG0114 isolates but failed to complement VCG0114 isolates, is included here as a representative isolate.

${ }^{\mathrm{z}}$ Controls consisted of plants inoculated with water with 10 replicates in each of the three trials.

Table 4. Fresh shoot weight, shoot height, percent leaves wilted, and vascular browning index of Gossypium hirsutum germplasm line Barbren 713 after 42 days of growth in soils infested with isolates of different genotypes within VCG0114 of Fusarium oxysporum f. sp. vasinfectum from diverse geographic origins ${ }^{\mathrm{u}}$

\begin{tabular}{lccccc}
\hline Type $^{\mathbf{v}}$ & No. of isolates & Shoot weight (g) & Shoot height (cm) & Percent leaves wilted $^{\mathbf{w}}$ & Vascular browning index $^{\mathbf{x}}$ \\
\hline NCh & 16 & $10.7(57.7)^{\mathrm{y}} \mathrm{a}$ & $35.2(23.8)^{\mathrm{y}} \mathrm{bc}$ & $71.2 \mathrm{a}$ & $69.9 \mathrm{a}$ \\
$\mathrm{MTCh}$ & 2 & $4.5(82.2) \mathrm{a}$ & $20.1(56.5) \mathrm{a}$ & $76.7 \mathrm{a}$ & $80.0 \mathrm{a}$ \\
$\mathrm{NChA}$ & 1 & $21.1(16.6) \mathrm{b}$ & $41.7(9.7) \mathrm{bc}$ & $8.6 \mathrm{~b}$ & $6.0 \mathrm{~b}$ \\
$\mathrm{TCa}$ & 13 & $7.7(69.6) \mathrm{a}$ & $23.6(48.9) \mathrm{a}$ & $58.9 \mathrm{a}$ & $65.5 \mathrm{a}$ \\
$\mathrm{MiTCa}$ & 1 & $22.1(12.6) \mathrm{b}$ & $41.4(10.4) \mathrm{bc}$ & $11.1 \mathrm{~b}$ & $18.0 \mathrm{~b}$ \\
$\mathrm{NCa}$ & 1 & $14.9(41.1) \mathrm{ab}$ & $26.7(42.2) \mathrm{ab}$ & $51.9 \mathrm{ab}$ & $44.0 \mathrm{ab}$ \\
Control $^{\mathrm{z}}$ & 0 & $25.3 \mathrm{~b}$ & $46.2 \mathrm{c}$ & $0.0 \mathrm{~b}$ & $0.0 \mathrm{~b}$ \\
\hline
\end{tabular}

u Values are the least squares means of the responses treated by the number of isolates indicated in the second column for each genotype of VCG0114 of F. oxysporum f. sp. vasinfectum with five replicates for each isolate in each of the two trials. Means were separated at $P=0.05$ using least squares means with the Tukey-Kramer adjustment. Means with the same letter within the column are not significantly different. The following isolates were used: CH1869, 1889, 1907 to 1909, 1911 to 1913, 1934, 1944, 1960, 1965, 1969, 1970, 1973, and 1975 for NCh; CH1971 and 1972 for MTCh; CH1967 for NChA; CA9, 14 , MD305 to 307,309 to 311, and 313 to 317 for TCa; MD308 for MiTCa; and MD312 for NCa.

$\checkmark$ Type: genotypes within VCG0114 were designated based on the presence of transposable elements within the $P H O$ gene in conjunction with their geographic origins. $\mathrm{NCh}=$ no transposon/China; $\mathrm{MTCh}=$ mutator-like transposable element $(M U L E)$ within Tfol transposon/China; NChA = no transposon/China-negative for $T f o 1$ insertion in the promoter region of $N A T ; \mathrm{TCa}=T f o 1$ transposon/California; $\mathrm{MiTCa}=$ miniature inverted-repeat transposable element $(M I T E)$ within Tfol transposon/California; and NCa = no transposon/California.

${ }^{w}$ Percentage of leaves wilted was calculated by dividing the number of wilted leaves including the leaf scars by the total number of leaves including the leaf scars within a wilted plant multiplied by 100 .

$x$ Vascular browning index was initially assigned with the following scale: $0=$ no staining; $1=0$ to $10 \%$ of cross section stained; $2=10$ to $30 \%$ of area stained; $3=$ 30 to $70 \%$ of area stained; $4=70$ to $90 \%$ of area stained; and 5 = 90 to $100 \%$ of area stained or the plant was dead. For ANOVA-type statistical analysis, they were converted to percentage basis by taking the midpoint for each scale: scale 0 became $0 \%, 1$ became $5 \%, 2$ became $20 \%, 3$ became $50 \%, 4$ became $80 \%$, and 5 became $95 \%$.

y Percent reduction compared with control is given in the parentheses.

${ }^{\mathrm{z}}$ Controls consisted of plants inoculated with water with 10 replicates in each of the two trials. 
Tgo polymerase mix produced 8 -kb amplicons. Sequencing with $P H O$ and Tfol primers (Ortiz et al. 2017a) gave identical sequences with that of California VCG0114 isolate CA9 (Fig. 1, left panel, T [Tfol] genotype) except for an inserted MULE sequence with a 9-bp
TSD and 92-bp TIR sequences in the Tfol at position $76 \mathrm{bp}$. The inserted MULEs were sequenced for the two isolates with new primers designed based on sequence homology to closely related MULEs and were found to be identical for the two isolates.

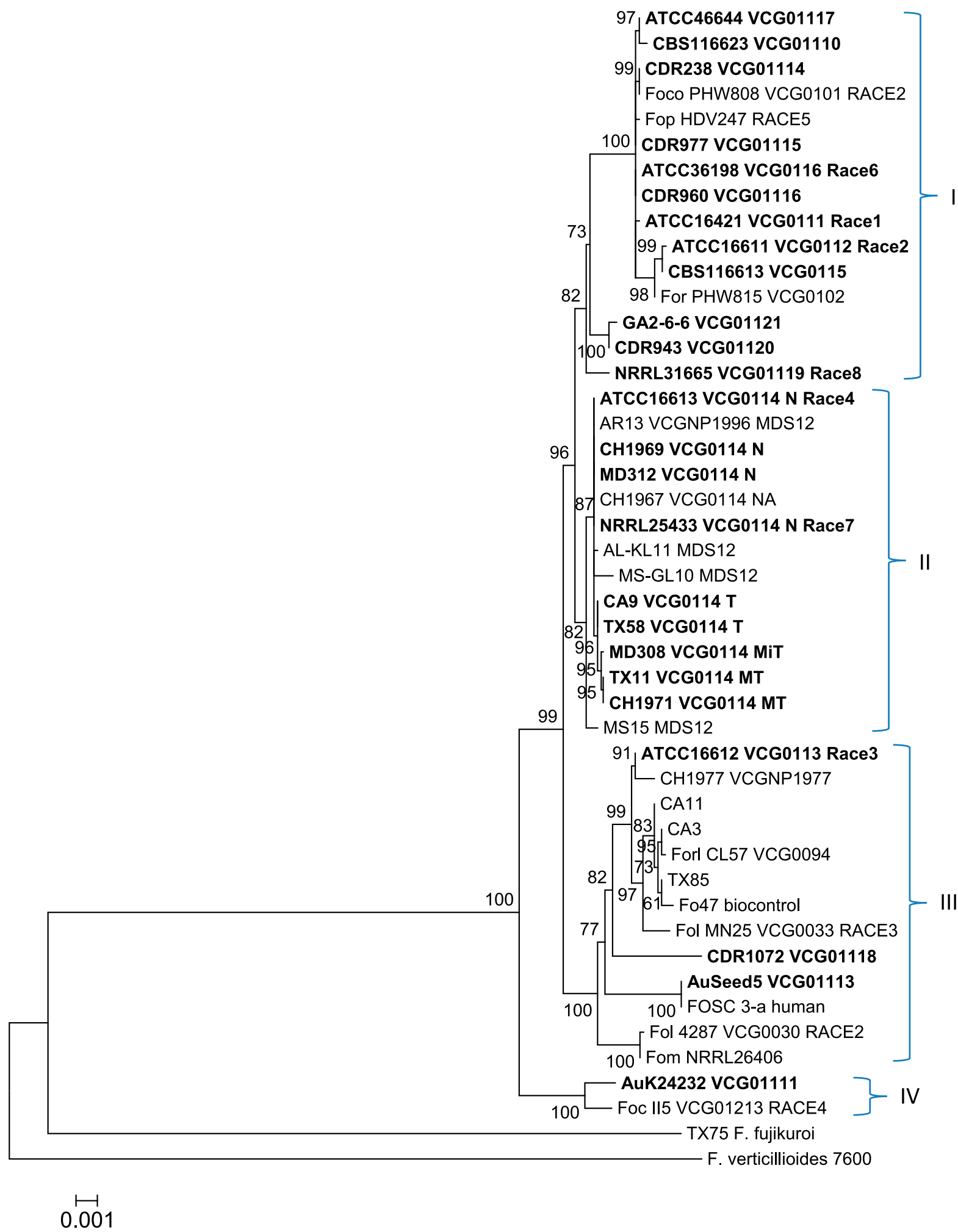

Fig. 2. Maximum likelihood phylogenetic tree of diverse Fusarium oxysporum isolates. The bold isolates are pathogenic to cotton. The tree was constructed using IQtree 1.6 .9 based on the concatenated sequences of translation elongation factor $1-\alpha$ ( $E F-1 \alpha, 1,831$ bp aligned length), phosphate permease (PHO, 1,945 bp aligned length), and $\beta$-tubulin ( $B T, 1,892 \mathrm{bp}$ aligned length) from $F$. oxysporum f. sp. vasinfectum isolates representing known vegetative compatibility groups and sequence types, and from $F$. oxysporum isolates associated with cotton. The Tfo1 insertion sequence is coded as a single character, and insertions of each MITE or MULE into the Tf01 are coded as additional single different characters in the PHO sequence. Sequences of a number of F. oxysporum formae speciales (pisi HDV247, conglutinans PHW808, raphani PHW815, lycopersici 4287, melonis NRRL26406, lycopersici MN25, radicis-lycopersici CL57, and cubense II5), a clinical F. oxysporum isolate FOSC 3-a, and F. oxysporum biocontrol agent Fo47, whose genome sequences are available from NCBI, were also included in the analysis as references. Sequences of $F$. fujikuroi isolate TX75 from cotton and $F$. verticillioides strain 7600 (Fv7600), were also included to root the tree. Best-fit models according to Bayesian information criterion were $\mathrm{TN}+\mathrm{F}+\mathrm{I}$ for $E F-1 \alpha, \mathrm{TN}+\mathrm{F}+\mathrm{G} 4$ for $P H O$, and $\mathrm{TIM} 2+\mathrm{F}+\mathrm{l}$ for $B T$. Ultrafast bootstrap frequencies from 1,000 replications are noted next to the branches. The scale is equivalent to 1 nucleotide substitution per 1,000 bp. 
F. oxysporum f. sp. vasinfectum isolates from 2017 West Texas sample collection. Stem samples of wilted cotton plants collected in fields of El Paso and Hudspeth counties of Texas in 2017 yielded a total of $69 F$. oxysporum isolates. Vegetative compatibility analysis confirmed that 66 of them belonged to $F$. oxysporum f. sp. vasinfectum VCG0114 (Table 1). Sequence analysis of $E F-1 \alpha, P H O$, and $B T$ genes from some of these 66 isolates showed them to be identical to either California VCG0114 isolate CA9 or China VCG0114 isolate CH1971 (genotype T [Tfol] or MT [MULE/Tfol], respectively, in Figure 1, left panel). Sequence analysis of the other three nonVCG0114 isolates indicated that they shared identical sequences and clustered in clade III as another new genotype in the phylogenetic tree (Fig. 2). These isolates were nonpathogenic to cotton (see below). Many nonpathogenic Fusarium spp. isolates (data not given) were obtained, but only one, TX75, was analyzed in this study. Sequence analysis identified this isolate as $F$. fujikuroi (Fig. 2). This isolate was also nonpathogenic to cotton (see below).

VCG0114-specific PCR detection method. To develop a VCG0114-specific PCR detection method, we searched for all the Tfol insertion events in the genome of the VCG0114 isolate NRRL25433 with $5^{\prime}$ TIR (CAGTGTGTCCATCAATCAAGT) and 3' TIR (CAGTGTGTCCATCAACCAAGT) of Tfol. Thirty-one loci were identified for each TIR, and only a fraction of the loci contained the full length Tfol gene with corresponding $5^{\prime}$ TIR and $3^{\prime}$ TIR. Many of the loci resided at the end of the sequence contigs. Sequences flanking Tfol were used to design primers for the 62 loci. $5^{\prime}$ or $3^{\prime}$ flanking primers were paired with the FovT-R or FovT-F primer (Fig. 1, left panel), respectively, to screen one or two isolates of each genotype listed in Table 1. The primer pair FovT-F and FovA-R (Fig. 1, left panel) consistently gave positive bands only for the VCG0114 isolates in this screen.

The specificity of the primer pair FovT-F and FovA-R was tested against a panel of 154 Fusarium isolates (Table 1; Supplementary Table S1, column FovT-A [500 bp]). All 110 VCG0114 (race 4) isolates, except one (CH1967), tested positive. Pathogenicity tests (see below) showed that this isolate is not pathogenic to cotton. None of the 28 isolates representing all other known VCGs and genotypes of F. oxysporum f. sp. vasinfectum tested positive. This PCR test also differentiated the eight MDS-12 isolates from VCG0114 isolates. These MDS-12 isolates had sequences in the $E F-1 \alpha, P H O$, and $B T$ loci that were closely related to those of VCG0114 (Fig. 2, clade II), with four of them (VCGNP1996) having DNA sequences identical with those of the VCG0114 reference isolate ATCC16613. The three $F$. oxysporum isolates and one $F$. fujikuroi isolate from the 2017 Texas collection and the four $F$. oxysporum isolates from 2015 China collection, all nonpathogenic to cotton, tested negative.

The loci containing these priming sites resided in the supercontig 975 (1,104 bp). Sequencing the flanking regions using primers based on sequence homology identified most of the Tfol sequence at the upstream flank and a NAT gene sequence at the downstream flank (Fig. 1, left panel). BLAST search using this region against the isolate NRRL25433 genome revealed the presence of two paralogues in the contigs of AGNC01000688 and AGNC01000674, respectively. This region $(5,847 \mathrm{bp})$ was sequenced from 15 representative VCG0114 isolates, and all had identical sequences with that of isolate NRRL25433. In each case, Tfol was inserted at the promoter region of the NAT gene at position $-659 \mathrm{bp}$. Limited attempts to sequence the orthologous copy of the region failed for a few closely related MDS-12 isolates such as AR13, which had identical $E F-1 \alpha$, $P H O$, and $B T$ sequences with that of Indian VCG0114 isolates or other VCG isolates such as GA2-1-6, although paralogous fragments were identified.

MITE insertion in the Tfol inserted in the $\mathrm{PHO}$ gene of VCG0114 isolate MD308. When the gel picture of the amplicon of the multiplex PCR test with primers FovP-F, FovT-R, and FovP-R (Ortiz et al. 2017a) for isolate MD308 was reexamined, the band was about $80 \mathrm{bp}$ larger than expected for the T genotype (Fig. 1, right panel top, lane MiT versus lane T). Sequencing the PHO gene for this isolate revealed an additional 71-bp nonautonomous MITE inserted at a position $38 \mathrm{bp}$ from the $5^{\prime}$ TIR of Tfol
(Fig. 1, left panel, MiT genotype). In comparison, the MULE is inserted at position $76 \mathrm{bp}$ of the Tfol in the $P H O$ of the China isolate CH1971 and Texas isolate TX11 (Fig. 1, left panel, MT genotype). The insertion position of Tfol in the $\mathrm{PHO}$ gene is the same as that found in other California isolates (Ortiz et al. 2017a), Texas isolates, and China isolate CH1971.

Genotype determination and detection of VCG0114 isolates. Altogether, four transposable element insertion variations were observed among VCG0114 isolates from diverse geographic origins. These events provided an opportunity to genotype the VCG0114 isolates and to elucidate their probable evolutionary relationships. Thus, the N (null), T (Tfol), MT (MULE/Tfo1), or MiT (MITE/Tfol) genotypes are designated for VCG0114 isolates without a transposable element insertion in the $\mathrm{PHO}$ gene, with a Tfol insertion in the $\mathrm{PHO}$ gene, with a MULE insertion in the inserted Tfol in the $P H O$ gene, or with a MITE insertion in the inserted Tfol in the $P H O$ gene, respectively (Fig. 1, left panel). Primer pair FovP-F and FovT-R (Ortiz et al. 2017a) was designed to specifically detect T (583-bp) and MiT (663-bp) genotypes, whereas primer pair FovP-F and FovM-R specifically detected the MT (426-bp) genotype. The N (396-bp) genotype was specifically detected by primer pair FovP-F and FovP-R following a positive test with primer pair FovT-F and FovA-R in the VCG0114-specific PCR test.

All 154 Fusarium isolates in the present study were tested using genotype-specific primer pairs (Table 1; supplementary materials). The tests were highly specific to the genotypes of VCG0114 isolates, and no false negatives or positives were observed. These tests can also be conveniently run in a single multiplex PCR with a primer mixture of FovP-F, FovP-R, FovT-R, and FovM-R. An example is given in Figure 1 (right panel top), which differentiated the four genotypes of VCG0114.

The proposed evolutionary relationships among VCG0114 genotypes are shown in Figure 1 (right panel bottom). Insertion of a $T f o l$ in the $\mathrm{N}$ genotype (first generation, arbitrary reference point) $\mathrm{PHO}$ gene generated the T genotype (second generation). Two independent insertion events, one with a MULE insertion at Tfol position of $76 \mathrm{bp}$ in the $P H O$ gene and the other with a MITE insertion at Tfol position of $38 \mathrm{bp}$ in the $P H O$ gene, gave rise to the MT (third generation) and MiT (third generation) genotypes, respectively. Further justifications for the proposed evolutionary path are presented in the Discussion section.

A limited number of historical VCG0114 isolates from the NRRL collection, six from India and two from China, belonged to the $\mathrm{N}$ genotype (Fig. 1, left panel; first generation; Table 1). Among 17 California isolates (2001 to 2014) analyzed, one belonged to the $\mathrm{N}$ genotype (first generation), 15 to the T genotype (second generation), and one to the MiT genotype (third generation). Among 19 China 2015 isolates, most isolates (17) belonged to the $\mathrm{N}$ genotype (first generation), and two (one county) to the MT genotype (third generation). For the 10 of $17 \mathrm{~N}$ genotype isolates whose host sources were known, six were from $G$. hirsutum cultivars and four from $G$. barbadense cultivars. In Texas (2017), 30 isolates from El Paso County belonged to the T genotype (second generation), which is the prevailing genotype in California. Twenty-eight isolates from El Paso County and eight from Hudspeth County belonged to the MT genotype (third generation), which also was found as a minor genotype in China (2015).

Pathogenicity. Pathogenicity of the four genotypes of VCG0114 isolates was assessed on G. barbadense cultivar Pima S-7 (Table 2), G. hirsutum cultivar ST 474 (Table 3), and G. hirsutum germplasm line Barbren 713 (Table 4). Soil-infestation assays in which the fungal inoculum is injected into soil without injury to the root system were utilized. The possible effect of geographic origins of each genotype on pathogenicity was also assessed. To alleviate complications arising from natural variations of virulence among isolates within each type, a large number, whenever available, of isolates in each type were included in the assay and mean responses for each type were used for the analysis. The number of isolates included in each type are given in Tables 2, 3, and 4 for assays on the three cotton hosts. 
Texas, China, and California collection isolates were included in the assay on Pima S-7 (Table 2). None of the three Texas F. oxysporum isolates (TX76, 84, and 85) that clustered with clade III in Figure 2 nor the $F$. fujikuroi isolate TX75 were pathogenic to cotton (Table 2, labeled as NPF). The VCG0114 isolate CH1967 from the 2015 China collection, which failed the VCG0114-specific PCR test, also was nonpathogenic (Table 2). The rest of the genotypes of VCG0114 from all geographic locations caused severe wilt symptoms, with the percentage of leaves wilted and the vascular browning indexes ranging from 57 to $93 \%$ and from 55 to $94 \%$, respectively (Table 2). Most of the inoculated plants were killed by the end of the trials, especially when inoculated with Texas isolates and California TCa and MiTCa isolates. These isolates also caused significant reductions in shoot weight (63 to $95 \%$ ) and shoot height (50 to $78 \%$ ) compared with controls. Except for shoot height, the values for percent shoot weight reduction, percent leaves wilted, and vascular browning index are very similar for a given genotype. For example, for the TTx genotype, the corresponding values were 92, 93, and $93 \%$, respectively, indicating their close relationships.

The Texas isolates were more virulent than were the 2015 China isolates, irrespective of their genotypes, in shoot weight reduction ( $>91$ versus $<75 \%$ ), shoot height reduction ( $>72$ versus $<55 \%$ ), percent leaves wilted ( $>91$ versus $<76 \%$ ), and vascular browning index ( $>92$ versus $<78 \%$ ); all differences, except for some comparisons of percent leaves wilted, were significant, $P=0.05$ (Table 2). The MT

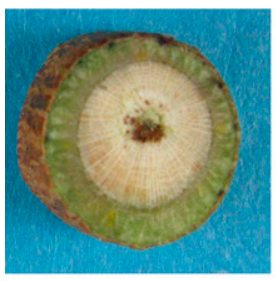

MTTx

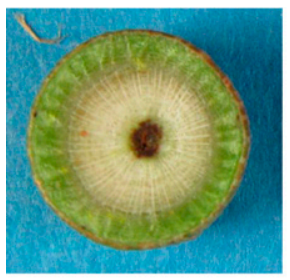

$\mathrm{NCh}$

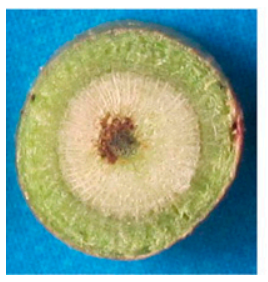

$\mathrm{TCa}$

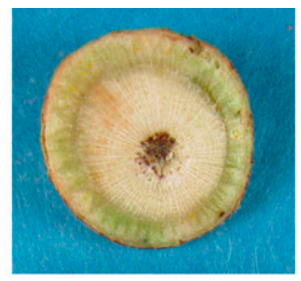

TTx

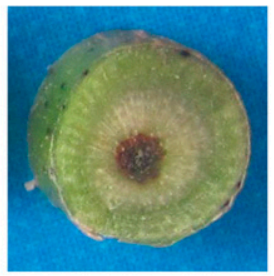

MTCh

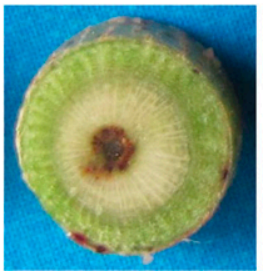

MiTCa

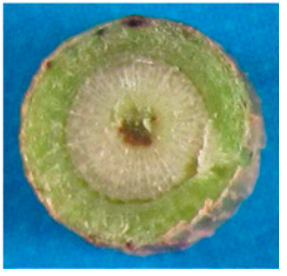

$\mathrm{NCa}$
Fig. 3. Typical discoloration of pith (central cylinder) and nearby primary xylem in plants that showed symptoms in cotyledons and lower leaves several weeks after inoculation. Top row: cross sections of plants of Gossypium hirsutum Barbren 713$32 \mathrm{BC}_{3}$ line with VCG0114 resistance introgressed from a resistant $G$. arboreum accession. After an initial 3 weeks of incubation at $23 / 18^{\circ} \mathrm{C}$, the temperature was increased to $28 / 22^{\circ} \mathrm{C}$ for 3 additional weeks to recover from the disease. Middle and bottom rows: cross sections from 6-week-old G. hirsutum Coker 312 plants kept at $23 / 18^{\circ} \mathrm{C}$. Plants were inoculated with the following VCG0114 genotype isolates: 2017 Texas isolates TX11 and TX39 for MTTx and TTx, respectively; 2015 China isolates $\mathrm{CH} 1969$ and $\mathrm{CH} 1971$ for NCh and MTCh, respectively; and California isolates (2001 to 2014) CA9, MD308, and MD312 for TCa, MiTCa, and $\mathrm{NCa}$, respectively. genotype isolates from Texas were more virulent than were the MT genotype isolates from China. There were no significant differences in any of the four response variables between the California and China isolates, irrespective of genotypes. The four response variables for the corresponding $\mathrm{N}$ genotypes in the China and California collections were almost identical. The T genotype in the Texas collection was significantly more virulent than was the corresponding genotype in the California collection in shoot weight reduction, percent leaves wilted, and vascular browning index response variables. No significant difference was observed in any response variable among the genotypes within each of the Texas, China, and California collections, except a significantly higher vascular staining index was observed for the N genotype compared with the MT genotype in the China collection.

Pathogenicity assays with $G$. hirsutum ST 474 were conducted with China and California collection isolates, China and Indian isolates from the NRRL collection, and an MDS-12 isolate AR13 (Table 3). The four F. oxysporum VCGNP1977 isolates from wilted cotton from the China collection were also included. Only the MT isolates from the China collection were able to cause severe wilt and to kill most of the inoculated plants, with significant reductions in shoot weight (81\%) and height $(61 \%)$ and significant increases in percentage of wilted leaves $(81 \%)$ and vascular browning $(86 \%)$ compared with water-treated control plants. The MTCh isolates also were significantly more virulent than were isolates of any other type in all disease response variables. The four plant response variables for the NCh, NCa, TCa, and MiTCa genotypes were not significantly different from each other, except percent leaves wilted was greater for NCh than for TCa. The reductions in shoot weight and shoot height and increases in the percent leaves wilted and the vascular browning index for the $\mathrm{NCh}, \mathrm{NCa}, \mathrm{TCa}$, and MiTCa genotypes were in the range of 16 to 32,15 to 23,24 to 46 , and 29 to $42 \%$, respectively, compared with water treatment controls. Not all these values were significant, but at least the vascular browning index remained significant for these genotypes in the two collections compared with controls. The isolates with the $\mathrm{N}$ genotype in the China and California collections were not significantly different from each other in virulence as measured by the four disease response variables. China isolate CH1967 (NChA genotype), China F. oxysporum VCGNP1977 isolates, China isolates ( $\mathrm{N}$ genotype) from NRRL collection, Indian isolates (N genotype), and MDS-12 isolate AR30 all failed to cause any significant change in any disease response variable compared with water treatment controls.

Pathogenicity to G. hirsutum Barbren 713 was assessed for VCG0114 isolates from the China and California collections (Table 4). The MT genotype isolates from China were the most virulent isolates and caused severe wilt and death of most of the inoculated plants, with significant reductions in shoot weight $(82 \%)$ and height $(57 \%)$ and significant increases in percentage of wilted leaves (77\%) and vascular browning (80\%) compared with the water-treated controls. The TCa isolates showed virulence similar to that of MTCh isolates. The China isolate CH1967 (NChA type) was nonpathogenic. The sole isolate with the MiT genotype, MD308, also failed to cause any significant change in any disease response variable. The $\mathrm{N}$ genotypes from the China and California collections ( $\mathrm{NCh}$ and $\mathrm{NCa}$ ) were not significantly different from each other in any of the disease response variables. Although the NCh genotype caused significant changes in disease response variables of shoot weight, percent leaves wilted, and vascular browning index compared with the control treatments, NCa caused significant change in shoot height only. Pairwise comparisons between the genotypes of N, T, and MT for any of the disease response variables showed significant differences between NCh versus MTCh and NCh versus TCa for shoot height only.

For assays on all three cotton hosts (Tables 2, 3, and 4), the value of percent leaves wilted was always very close to the value of the corresponding vascular browning index for each genotype. When the wilted leaves and the adjunct stem sites were examined, darkly stained pith (central cylinder) of the adjunct stem was often observed. 
Staining of pith and adjoining primary xylem, instead of secondary and primary xylem, is characteristic of VCG0114 isolates (Fig. 3).

\section{Discussion}

Based on the various insertions of Tfol, MULE, and MITE into the PHO locus (Fig. 1, left panel) of the VCG0114 isolates, we divided these isolates into four genotypes and developed PCR methods to specifically detect each genotype. We also developed a VCG0114specific PCR detection method based on the Tfol insertion event at the NAT gene locus. The T, MT, and MiT genotype determinations were highly specific without any false positives or negatives, and the VCG0114-specific PCR test had only one false negative out of 110 VCG0114 isolates and no false positives. The specificity of the $\mathrm{N}$ genotype determination is contingent upon a positive result with the VCG0114-specific PCR test. The VCG0114-specific PCR detection method can discriminate "race 4-like" MDS-12 isolates from Mid-South states, which have $E F-1 \alpha, P H O$, and $B T$ sequences identical that those of isolates of the $\mathrm{N}$ genotype. This method provides an alternative approach to vegetative compatibility analysis, which is labor intensive and time consuming. However, pathogenicity tests should never be by-passed in any new field studies.

Dividing the VCG0114 isolates into four genotypes allowed us to trace their evolutionary relationships. This evolutionary relationship is straightforward, as shown in Figure 1 (right panel bottom), unless the inserted transposable element in the $\mathrm{PHO}$ locus reverts to the exact original noninserted state with perfect excision. Perfect excision is a rare event for class II (DNA) transposons, to which these three transposons belong (Lisch 2015).

Upon movement of these elements by the "cut and paste" mechanism, the double-stranded break at the donor site is repaired either by homologous recombination or by nonhomologous end-joining (Feschotte and Pritham 2007; Lisch 2015; Raizada et al. 2001). Homologous recombination repair results in the recreation of the original nonexcised donor site with the transposable element still in place. Nonhomologous repair of the excision sites is seldom perfect and often leaves behind a transposon footprint with deletions, additions, and filler elements (Feschotte and Pritham 2007; Lisch 2015; Raizada et al. 2001). Therefore, examination of the insertion sites for footprints should reveal whether a reversion had occurred. Sequence analysis near Tfol insertion position 462 bp in the $P H O$ gene from 20 isolates of the $\mathrm{N}$ genotype revealed no variation among them (Ortiz et al. 2017a). The sequences also aligned perfectly with $P H O$ sequences from other known VCGs and showed no indels around position $462 \mathrm{bp}$. The presented sequences at this position indicate no reversion and firmly establish the $\mathrm{N}$ genotype as the founding (arbitrary reference point in our study) genotype. This genotype occurs in $100 \%$ of historical isolates from India and China in the NRRL collection, $6 \%$ of isolates from California, $84 \%$ of isolates from China in 2015 , and $0 \%$ of isolates from Texas.

Sequence analysis near the MULE insertion position at $76 \mathrm{bp}$ from $5^{\prime}$ TIR of Tfol in the $P H O$ gene from 12 isolates of genotype $\mathrm{T}$ or MiT also revealed no variation among them (Ortiz et al. 2017a). The sequences aligned perfectly with other Tfol genes from NCBI, and sequences at this position support no reversion. Sequence analysis near MITE insertion position $38 \mathrm{bp}$ from $5^{\prime}$ TIR of Tfol in the $\mathrm{PHO}$ gene from 18 isolates of genotype $\mathrm{T}$ and MT also revealed no variation among them (Ortiz et al. 2017a). The sequences also aligned perfectly with other Tfol genes available at NCBI. Sequences at this position also support no reversion. Thus, the proposed evolutionary relationship in Figure 1 (right panel bottom) is strongly supported. This relationship is also consistent with the maximum likelihood phylogenetic analysis depicted in clade II of Figure 2. The $\mathrm{T}$ genotype (second generation) was found to comprise 88 and $45 \%$ of VCG0114 isolates from California and Texas collections, respectively, but was not found in other collections. The MT genotype (third generation) was found to comprise 11 and 55\% of VCG0114 isolates from China and Texas collections, respectively, but was not found in other collections. Only one isolate, California VCG0114 isolate MD308, belongs to the MiT genotype (third generation).
Sharing of certain identical genotypes between or among different collections suggests movement of the pathogen by seed. Transmission of $F$. oxysporum f. sp. vasinfectum by cottonseed has been reported (Bennett et al. 2008; Davis et al. 2006; Liu et al. 2011; Taubenhaus and Ezekiel 1932). The host also may alter genotype distribution within a field. In our pathogenicity tests, significantly greater virulence of the MT (minor) over the $\mathrm{N}$ (major) genotype of the China collection was observed on ST 474 but not on Pima S-7 and Barbren 713 (Table 3 versus Tables 2 and 4). Therefore, continued cultivation of certain $G$. hirsutum cultivars such as ST 474 may favor the buildup of the MT genotype population while suppressing the $\mathrm{N}$ genotype population. Susceptible cultivars favor rapid buildup of inoculum and expansion of disease foci, whereas resistant or intermediate resistant cultivars favor seed transmission of the pathogen. Introduced together from an infested area, they may accelerate disease establishment in a new location.

The occurrence of the MT genotype both in Texas and China could arise from independent MULE insertion events, but that is highly unlikely, as will be discussed below. Although class II transposons preferentially insert into functional genes, there is little specificity in insertion site sequence requirements (Raizada et al. 2001). When the class II transposon Tfol moves to a new position, it duplicates the 8-bp host DNA sequence at the insertion site. Therefore, the TSD sequences will flank the newly introduced Tfol. Analysis of both the $5^{\prime}$ and $3^{\prime}$ TSD sequences of Tfol in the genome of VCG0114 isolate NRRL25433 revealed that there were no more than two identical sequences for each TSD, indicating no target site preference. Therefore, the probability of independent insertions exactly at the same base position within the whole genome to generate the MT genotype in both El Paso and in China was nearly zero. They are probably from the same source. The most diverse genotypes of VCG0114 were expected at the center of the origin of VCG0114 (race 4) in India. However, the six historical VCG0114 isolates from India all belonged to the $\mathrm{N}$ genotype. Determining the distribution of VCG0114 genotypes in various countries may provide clues as to their origin and movement.

Most of China VCG0114 isolates belong to the N genotype, including the two historical race 7 isolates deposited in the NRRL collection and 17 of 19 putative race 7 isolates in our China collection. The two MT genotype isolates from Shawan County in Northern Xinjiang may be a new introduction into China. This is consistent with the fact that no T genotype, the intermediate genotype between $\mathrm{N}$ and MT (Fig. 1, right panel bottom), was detected in the China collection and the distribution of the MT genotype was limited to one county only.

The VCG0114 isolate CH1967 (NChA type) that failed the VCG0114-specific PCR detection was not pathogenic to cotton (Tables 2, 3, and 4). It was able to grow in rich media such as PDA but failed to grow in minimal media such as Czapek-Dox agar, indicating that the nutrient assimilation pathway was defective. This may be caused by the unusually high class II DNA transposon activity in VCG0114 isolates. The genome of VCG0114 isolate NRRL25433 harbored more than 62 TIR of Tfol, whereas each genome of 10 other $F$. oxysporum forma specialis isolates sequenced by the Broad Institute (available from NCBI) contained fewer than six TIR of Tfol, except for PHW815, which contained 18 TIR of Tfol. Copy numbers of transposases corresponding to Tfo1, MULE, and MITE in VCG0114 isolates generally were at least 10 -fold higher than copy numbers in other $F$. oxysporum $\mathrm{f}$. sp. vasinfectum VCG isolates (Ortiz et al. 2017b). The proliferation of these class II transposon activities not only deactivates functional genes but also may modulate gene activities by inserting into the promoters of genes. For example, the Tfol insertion into the promoter region of the NAT gene, a gene that may have function in chromatin modification and gene regulation, may reprogram the NAT gene expression pattern. The loss of virulence of the historical Indian (including ATCC 16613) and Chinese VCG0114 isolates from the NRRL collection in our pathogenicity assay may also be owing to the activity of these class II transposons during long-term storage without host selective pressures. Kim 
et al. (2005) also reported that ATCC16613, the race 4 reference isolate, failed to cause any disease symptoms in cotton. Controlled class II transposon activity may have enabled VCG0114 isolates to gain host specificity to cotton or to convert the isolates from a commensal to a pathogenic fungus. Introducing a transposon-rich chromosome from $F$. oxysporum f. sp. lycopersici into a biocontrol isolate of $F$. oxysporum converted the latter to a pathogen (Ma et al. 2010).

We tried to resolve the issue of whether race 4 and race 7 are the same. Chen et al. (1985) designated a group of $F$. oxysporum isolates from cotton in China as race 7 because they attacked $G$. hirsutum, $G$. barbadense, and G. arboreum, whereas Armstrong and Armstrong (1960) reported that race 4 was avirulent to G. hirsutum and G. barbadense but attacked G. arboreum 'Rozi'. However, the differential hosts used by Chen et al. were not the same as those used by Armstrong and Armstrong, and the inoculation methods also differed. Our pathogenicity tests directly compared race 4 isolates from California and Texas with putative race 7 isolates from China (2015). All isolates, except one isolate from China, caused disease in a soil-infestation assay without nematodes and thus belong to the nematode-independent pathotype, although some virulence variation exists among isolates (Tables 2, 3, and 4). Texas isolates were significantly more virulent than isolates from China and California on $G$. barbadense Pima S-7. No differences in pathogenicity were observed between isolates from the California collection and the China collection on Pima S-7; on G. hirsutum ST 474 or Barbren 713, the virulence of the major genotypes was similar. Furthermore, all China, California, and Texas collection isolates complemented each other in our vegetative compatibility analyses, and all complemented the original VCG0114 Indian reference isolate ATCC16613. They all shared identical $E F-1 \alpha, P H O$, and $B T$ sequences, except for the inserted transposable elements. These data show that there is no difference between race 4 and race 7 , and both races can be designated as VCG0114.

VCG0114 isolates belong to the nematode-independent (root-rot or vascular incompetent) pathotype. Isolations of $F$. oxysporum $\mathrm{f}$. sp. vasinfectum from the wilted plants in both Texas and China collections yielded a single pathogenic VCG regardless of their host being G. barbadense or G. hirsutum. This was in sharp contrast to the occurrence of multiple VCGs even from individual plants grown in fields infested with VCGs of the nematode-dependent (vascular competent) pathotype. In Georgia, wilted plants from a single field were attacked by two to five VCGs (Bell et al. 2017). Many of the individual plants were attacked by two and, in rare cases, three VCGs. Whether the recovery of only a single VCG from wilted plants of VCG0114-infested fields in Texas and China was owing to its strong competitiveness or owing to its soil type preference requires further elucidation.

Three and four $F$. oxysporum isolates other than VCG0114 were also identified from the infected cotton tissues in the Texas and China collections, respectively. None of them were pathogenic in our tests. Vegetative compatibility analyses of the four China isolates indicated they were compatible with each other but not with any other known F. oxysporum $\mathrm{f}$. sp. vasinfectum $\mathrm{VCG}$. These seven isolates belonged to clade III, where the nematode-independent pathotype VCG0113 isolate ATCC16612 resided (Fig. 2). California isolates CA3 and CA11, which were designated as race 3 by Kim et al. (2005), also belonged to this clade (Fig. 2), but they were more distantly related to VCG0113 than the four China nonpathogenic isolates. Although CA 3 and CA1 1 were recognized as race 3 based on DNA sequences, they were vegetatively incompatible with the VCG0113 (race 3) isolates and nonpathogenic to cotton (Cianchetta et al. 2015; Holmes et al. 2009; Kim et al. 2005; Ortiz et al. 2017a). Thus, clade III harbors many $F$. oxysporum isolates nonpathogenically associated with cotton. In addition, plants inoculated with these nonpathogenic isolates showed no characteristic pith darkening, which was observed for plants infected by VCG0113 or VCG0114 (Fig. 3).

In summary, this study demonstrated that all VCG0114 isolates, regardless of their origin, can cause disease without nematodes in the soil-infestation assay, and these isolates are especially virulent to Pima S-7. The PCR methods developed to specifically detect VCG0114 and the currently known four genotypes of VCG0114 will provide researchers, extension agents, and farmers a tool to genotype these isolates anywhere in the world. With the genotypes being determined on a global scale, not only can the future movement of these pathogens be traced, but past movement of isolates may be determined. This will help to control the further spread of this devasting pathogen and aid in breeding for resistance.

\section{Acknowledgments}

We thank R. M. Davis and H. K. Doan, University of California, Davis, for providing fungal isolates with CA or MD designations and MDS-12 isolates; T. S. Isakeit and J. E. Woodward, Texas A\&M University, for providing July 2017 Texas diseased cotton stem sample; and the USDA Agricultural Research Service Culture Collection (NRRL) for access to their culture collections.

\section{Literature Cited}

Abo, K., Klein, K. K., Edel-Hermann, V., Gautheron, N., Traore, D., and Steinberg, C. 2005. High genetic diversity among strains of Fusarium oxysporum f. sp. vasinfectum from cotton in Ivory Coast. Phytopathology 95: 1391-1396.

Armstrong, G. M., and Armstrong, J. K. 1960. American, Egyptian, and Indian cotton-wilt Fusaria: Their pathogenicity and relationship to other wilt Fusaria. Tech. Bull. U.S. Dep. Agric. 1219:1-19.

Armstrong, G. M., and Armstrong, J. K. 1978. A new race (race 6) of the cottonwilt Fusarium from Brazil. Plant Dis. Rep. 62:421-423.

Bell, A. A., Kemerait, R. C., Ortiz, C. S., Prom, S., Quintana, J., Nichols, R. L., and Liu, J. 2017. Genetic diversity, virulence, and Meloidogyne incognita interactions of Fusarium oxysporum isolates causing cotton wilt in Georgia. Plant Dis. 101:948-956.

Bell, A. A., Liu, J., Ortiz, C. S., Quintana, J., Stipanovic, R. D., and Crutcher, F. K. 2016. Population structure and dynamics among Fusarium oxysporum isolates causing wilt of cotton. Pages 153-158 in: Proceedings of Beltwide Cotton Conferences. S. Boyd and M. Huffman, eds. National Cotton Council of America, Memphis, TN.

Bell, A. A., Robinson, A. F., Quintana, J., Duke, S. E., Starr, J. L., Stelly, D. M., Zheng, X. T., Prom, S., Saladino, V., Gutierrez, O. A., Stetina, S. R., and Nichols, R. L. 2015. Registration of BARBREN-713 germplasm line of upland cotton resistant to reniform and root-knot nematodes. J. Plant Regist. 9:89-93.

Bennett, R. S., Hutmacher, R. B., and Davis, R. M. 2008. Seed transmission of Fusarium oxysporum f. sp. vasinfectum race 4 in California. J. Cotton Sci. 12:160-164.

Bennett, R. S., Scott, T. Z., Lawrence, K. S., and Lawrence, G. W. 2013. Sequence characterization of race 4-like isolates of Fusarium oxysporum from Alabama and Mississippi. J. Cotton Sci. 17:125-130.

Chen, Q., Ji, X., and Sun, W. 1985. Identification of races of cotton wilt Fusarium in China. Sci. Agric. Sin. 6:1-6.

Chernomor, O., von Haeseler, A., and Minh, B. Q. 2016. Terrace aware data structure for phylogenomic inference from supermatrices. Syst. Biol. 65: 997-1008.

Cianchetta, A. N., Allen, T. W., Hutmacher, R. B., Kemerait, R. C., Kirkpatrick, T. L., Lawrence, G. W., Lawrence, K. S., Mueller, J. D., Nichols, R. L., Olsen, M. W., Overstreet, C., Woodward, J. E., and Davis, R. M. 2015. Survey of Fusarium oxysporum f. sp. vasinfectum in the United States. J. Cotton Sci. 19:328-336.

Davis, R. D., Moore, N. Y., and Kochman, J. K. 1996. Characterisation of a population of Fusarium oxysporum $\mathrm{f}$. sp. vasinfectum causing wilt of cotton in Australia. Aust. J. Agric. Res. 47:1143-1156.

Davis, R. M., Colyer, P. D., Rothrock, C. S., and Kochman, J. K. 2006. Fusarium wilt of cotton: Population diversity and implication for management. Plant Dis. 90:692-703.

Di Pietro, A., Madrid, M. P., Caracuel, Z., Delgado-Jarana, J., and Roncero, M. I. G. 2003. Fusarium oxysporum: Exploring the molecular arsenal of a vascular wilt fungus. Mol. Plant Pathol. 4:315-325.

Fahmy, T. 1927. The Fusarium disease (wilt) of cotton and its control. Phytopathology 17:749-767.

Fernandez, D., Assigbetse, K., Dubois, M. P., and Geiger, J. P. 1994. Molecular characterization of races and vegetative compatibility groups in Fusarium oxysporum f. sp. vasinfectum. Appl. Environ. Microbiol. 60:4039-4046.

Feschotte, C., and Pritham, E. J. 2007. DNA transposons and the evolution of eukaryotic genomes. Annu. Rev. Genet. 41:331-368.

Garber, R. H., Jorgenson, E. C., Smith, S., and Hyer, A. H. 1979. Interaction of population-levels of Fusarium oxysporum f. sp. vasinfectum and Meloidogyne incognita on cotton. J. Nematol. 11:133-137.

Gordon, T. R., and Martyn, R. D. 1997. The evolutionary biology of Fusarium oxysporum. Annu. Rev. Phytopathol. 35:111-128.

Halpern, H. C., Bell, A. A., Wagner, T. A., Liu, J., Nichols, R. L., Olvey, J., Woodward, J. E., Sanogo, S., Jones, C. A., Chan, C. T., and Brewer, M. T. 2018. First report of Fusarium wilt of cotton caused by Fusarium oxysporum f. sp. vasinfectum race 4 in Texas, U.S.A. Plant Dis. 102:446. 
Hoang, D. T., Chernomor, O., von Haeseler, A., Minh, B. Q., and Vinh, L. S. 2018. UFBoot2: Improving the ultrafast bootstrap approximation. Mol. Biol. Evol. 35:518-522.

Holdeman, Q. L., and Graham, T. W. 1954. Effect of the sting nematode on expression of Fusarium wilt in cotton. Phytopathology 44:683-685.

Holmes, E. A., Bennett, R. S., Spurgeon, D. W., Colyer, P. D., and Davis, R. M. 2009. New genotypes of Fusarium oxysporum f. sp. vasinfectum from the Southeastern United States. Plant Dis. 93:1298-1304.

Ibrahim, F. M. 1966. A new race of cotton-wilt Fusarium in the Sudan Gezira. Emp. Cotton Grow. Rev. 43:296-299.

Jorgenson, E. C., Hyer, A. H., Garber, R. H., and Smith, S. N. 1978. Influence of soil fumigation on Fusarium-root-knot nematode disease complex of cotton in California. J. Nematol. 10:228-231.

Kalyaanamoorthy, S., Minh, B. Q., Wong, T. K. F., von Haeseler, A., and Jermiin, L. S. 2017. ModelFinder: Fast model selection for accurate phylogenetic estimates. Nat. Methods 14:587-589.

Katan, J., Fishler, G., and Grinstein, A. 1983. Short-term and long-term effects of soil solarization and crop sequence on Fusarium wilt and yield of cotton in Israel. Phytopathology 73:1215-1219.

Katan, T., and Di Primo, P. 1999. Current status of vegetative compatibility groups in Fusarium oxysporum: Supplement (1999). Phytoparasitica 27:273-277.

Kim, Y., Hutmacher, R. B., and Davis, R. M. 2005. Characterization of California isolates of Fusarium oxysporum f. sp. vasinfectum. Plant Dis. 89:366-372.

Komada, H. 1975. Development of a selective medium for quantitative isolation of Fusarium oxysporum from natural soil. Rev. Plant Prot. Res. 8:114-124.

Kulkarni, G. S. 1934. Studies in the wilt disease of cotton in the Bombay Presidency. Indian J. Agric. Sci. 4:976-1048.

Lisch, D. 2015. Mutator and MULE transposons. Microbiol. Spectr. 3:1-24.

Liu, J., Bell, A. A., Wheeler, M. H., Stipanovic, R. D., and Puckhaber, L. S. 2011. Phylogeny and pathogenicity of Fusarium oxysporum isolates from cottonseed imported from Australia into California for dairy cattle feed. Can. J. Microbiol. 57:874-886.

Ma, L. J., van der Does, H. C., Borkovich, K. A., Coleman, J. J., Daboussi, M. J., Di Pietro, A., Dufresne, M., Freitag, M., Grabherr, M., Henrissat, B., Houterman, P. M., Kang, S., Shim, W. B., Woloshuk, C., Xie, X., Xu, J. R., Antoniw, J., Baker, S. E., Bluhm, B. H., Breakspear, A., Brown, D. W., Butchko, R. A., Chapman, S., Coulson, R., Coutinho, P. M., Danchin, E. G., Diener, A., Gale, L. R., Gardiner, D. M., Goff, S., Hammond-Kosack, K. E., Hilburn, K., Hua-Van, A., Jonkers, W., Kazan, K., Kodira, C. D., Koehrsen, M., Kumar, L., Lee, Y. H., Li, L., Manners, J. M., Miranda-Saavedra, D., Mukherjee, M., Park, G., Park, J., Park, S. Y., Proctor, R. H., Regev, A., Ruiz-Roldan, M. C., Sain, D., Sakthikumar, S., Sykes, S., Schwartz, D. C.,
Turgeon, B. G., Wapinski, I., Yoder, O., Young, S., Zeng, Q., Zhou, S. Galagan, J., Cuomo, C. A., Kistler, H. C., and Rep, M. 2010. Comparative genomics reveals mobile pathogenicity chromosomes in Fusarium. Nature 464:367-373.

Neal, D. C. 1954. The reniform nematode and its relationship to the incidence of Fusarium wilt of cotton at Baton Rouge, Louisiana. Phytopathology 44: 447-450.

Nelson, P. E., Toussoun, T. A., and Marasas, W. F. O. 1983. Fusarium Species: An Illustrated Manual for Identification. Pennsylvania State University Press, University Park, PA.

Nguyen, L. T., Schmidt, H. A., von Haeseler, A., and Minh, B. Q. 2015. IQ-TREE: A fast and effective stochastic algorithm for estimating maximum-likelihood phylogenies. Mol. Biol. Evol. 32:268-274.

Ortiz, C. S., Bell, A. A., Magill, C. W., and Liu, J. 2017a. Specific PCR detection of Fusarium oxysporum f. sp. vasinfectum California race 4 based on a unique Tfol insertion event in the PHO gene. Plant Dis. 101:34-44.

Ortiz, C. S., Magill, C. W., Bell, A. A., and Liu, J. 2017b. Characterization of phosphate permease in Fusarium oxysporum f. sp. vasinfectum: Growth, virulence and secondary metabolism. Pages 163-167 in: Proceedings of Beltwide Cotton Conferences. S. Boyd and M. Huffman, eds. National Cotton Council of America, Memphis, TN.

Raizada, M. N., Nan, G. L., and Walbot, V. 2001. Somatic and germinal mobility of the RescueMu transposon in transgenic maize. Plant Cell 13: 1587-1608.

Skovgaard, K., Nirenberg, H. I., O’Donnell, K., and Rosendahl, S. 2001. Evolution of Fusarium oxysporum f. sp. vasinfectum races inferred from multigene genealogies. Phytopathology 91:1231-1237.

Smith, S. N., and Snyder, W. C. 1975. Persistence of Fusarium oxysporum f. sp. vasinfectum in fields in absence of cotton. Phytopathology 65:190-196.

Snyder, W. C., and Hansen, H. N. 1940. The species concept in Fusarium. Am. J. Bot. 27:64-67.

Taubenhaus, J. J., and Christenson, L. D. 1936. Role of insects in the distribution of cotton wilt caused by Fusarium vasinfectum. J. Agric. Res. 53:703-712.

Taubenhaus, J. J., and Ezekiel, W. N. 1932. Seed transmission of cotton wilt Science 76:61-62.

Thompson, J. D., Higgins, D. G., and Gibson, T. J. 1994. Clustal-W-Improving the sensitivity of progressive multiple sequence alignment through sequence weighting, position-specific gap penalties and weight matrix choice. Nucleic Acids Res. 22:4673-4680.

Wang, C., and Roberts, P. A. 2006. A Fusarium wilt resistance gene in Gossypium barbadense and its effect on root-knot nematode-wilt disease complex. Phytopathology 96:727-734. 